\title{
Discovery of ovarian cancer biomarkers in serum using NanoLC electrospray ionization TOF and FT-ICR mass spectrometry
}

\author{
H. Robert Bergen, III ${ }^{\mathrm{a}, \mathrm{b}}$, George Vasmatzis ${ }^{\mathrm{c}}$, William A. Cliby ${ }^{\mathrm{d}}$, Kenneth L. Johnson ${ }^{\mathrm{a}, \mathrm{b}}$, \\ Ann L. Oberg ${ }^{\mathrm{e}}$ and David C. Muddiman ${ }^{\mathrm{a}, \mathrm{b}, *}$ \\ ${ }^{a}$ W.M. Keck FT-ICR Mass Spectrometry Laboratory, Mayo Proteomics Research Center, Rochester, MN, USA \\ ${ }^{\mathrm{b}}$ Department of Biochemistry and Molecular Biology, Mayo Clinic College of Medicine, Rochester, MN, USA \\ ${ }^{\mathrm{c}}$ Division of Experimental Pathology, Mayo Clinic College of Medicine, Rochester, MN, USA \\ ${ }^{\mathrm{d}}$ Department of Obstetrics and Gynecology, Mayo Clinic College of Medicine, Rochester, MN, USA \\ ${ }^{\mathrm{e}}$ Division of Biostatistics, Mayo Clinic College of Medicine, Rochester, MN 55905, USA
}

\begin{abstract}
Treatment of cancer patients is greatly facilitated by detection of the cancer prior to metastasis. One of the obstacles to early cancer detection is the lack of availability of biomarkers with sufficient specificity. With modern differential proteomic techniques, the potential exists to identify high specificity cancer biomarkers. We have delineated a set of protocols for the isolation and identification of serum biomarkers for ovarian cancer that exist in the low molecular weight serum fraction. After isolation of the low molecular weight fraction by ultrafiltration, the potential biomarkers are separated by reversed phase nano liquid chromatography. Detection via TOF or FT-ICR yields a data set for each sample. We compared stage III/IV ovarian cancer serum with postmenopausal age-matched controls. Using bioinformatics tools developed at Mayo, we normalized each sample for intensity and chromatographic alignment. Normalized data sets are subsequently compared and potential biomarkers identified. Several candidate biomarkers were found. One of these contains the sequence of fibrinopeptide-A known to be elevated in many types of cancer including ovarian cancer. The protocols utilized will be examined and would be applicable to a wide variety of cancers or disease states.
\end{abstract}

Keywords: Ovarian cancer, biomarkers, bioinformatics, serum, FT-ICR, TOF, mass spectrometry

\section{Introduction}

A $20 \%$ survival rate is expected for the $80 \%$ of women who present with advanced stage ovarian cancer (III/IV) while the survival rate for those detected at early stage is $>90 \%[8,10]$. Clearly, the most effective strategy for improving survival will be the development of reliable biomarkers for the detection of early stage ovarian cancer. Shifting the $80 \%$ of women who

*Corresponding author: David C. Muddiman, Ph.D., Medical Science Building 3-115, Mayo Clinic College of Medicine, 200 First Street, SW, Rochester, MN 55905, USA. Tel.: +1 507284 1997; Fax: +1 507284 9261; E-mail: muddiman.david@mayo.edu. present at disease stage III/IV into stages I/II would radically change the prognosis for women with ovarian cancer.

Survival rates have improved with the early detection of other cancers (e.g. breast, uterine, cervix $[8,10]$. One of the major contributing factors to the shift in stage from late to early presentation for breast and cervical cancer has been widely applied screening tests for these cancers. Unfortunately, ovarian cancer is often diagnosed in the advanced stages because of 1) lack of early warning symptoms, 2) lack of a reliable screening test and 3) lack of an easily performed diagnostic test for women with early symptoms. Despite intense research, little is known about the risk factors for ovar- 
ian cancer except for its association with family history and age $[11,12]$.

The average age-adjusted ovarian cancer incidence rate per 100,000 in 1996-2000 was $11.2(0.01 \%)$ and $56.3(0.06 \%)$ for individuals $<65$ and $>65$ years old, respectively [20]. The low incidence rate of $0.01 \%$ requires that any ovarian cancer biomarker have a positive predictive value (PPV, proportion of patients with positive test results who are correctly diagnosed) of $\sim 10 \%$ in order that one cancer patient will be found for each ten patients subjected to an invasive procedure [15]. The low prevalence of ovarian cancer requires that any ovarian biomarker(s) have a specificity (the ability to generate a true negative) of $99.9 \%$ with a sensitivity (proportion of true positives) of at least $67 \%$.

Currently the most widely used biomarker for ovarian cancer is carbohydrate (cancer) antigen 125 (CA 125 ) with a specificity which approaches $99 \%$ and a sensitivity of 50-60\% for early disease giving it a PPV of only $0.83-0.99 \%$ at the $0.01 \%$ incidence level for early disease and 4.76-5.66\% at the incidence level of those $>65$ years old [1]. These PPV values are far lower than the required PPV of $\sim 10 \%$ to even contemplate its use for population wide screening [15]. As such its use in screening is restricted to women at extremely high risk of developing ovarian cancer (familial cancer syndromes members).

Even though the search for a biomarker has been conducted in earnest for over 20 years, CA-125 was developed in the early 1980's primarily as a prognostic indicator for patients undergoing treatment for the disease [2]. This marker, along with the more recent marker OVX1, were both generated by screening monoclonal antibodies generated by immunizing mice with tumor cell homogenates. Selection of antibodies that did not cross react with noncancer cell lines afforded antibodies with tumor antigen specificity $[2,25]$. It has only been in the last two years that the CA-125 antigen has been identified as a new mucin $[13,26]$.

Unlike the indirect discovery of CA-125 and OVX1, the development of global technologies has caused a paradigm shift in biomarker discovery with the advent of comparative proteomic approaches directed toward disease biomarker discovery. Using a differential proteomics approach, the tissue or fluid from control and disease states is compared directly. Biomarkers that differentiate cancer from control are targeted for further evaluation and can be identified. There are numerous differential proteomics approaches that largely utilize either matrix assisted laser desorption/ionization (MALDI) or electrospray ioniza- tion (ESI) coupled to mass spectrometers. Techniques for examining intact proteins (high molecular weight) or protein fragments (low molecular weight) include surface-enhanced laser desorption and ionization timeof-flight (SELDI-ToF) [19] and multidimensional protein identification technology (MudPIT) [23,24]. Two such methods for conducting relative quantification (e.g., the comparison of a disease and a control sample) at the proteolytic fragment level include global internal standardization technology (GIST) [4] and isotopecoded affinity tagging (ICAT) [9].

Techniques that combine samples after differential labeling (e.g. ICAT, GIST) compare cancer and control samples in a pairwise fashion. The labeled sample acts as a de facto internal standard as part of the comparison protocol. Subsequent to the successful labeling of both samples, the control and cancer samples are pooled. It is this pooled sample that undergoes analysis and from which ratios are obtained that indicate the presence or absence of candidate biomarkers in the cancer sample relative to the control. Samples analyzed in this manner nullify variability due to retention time differences and subtle differences in ionization efficiency because the analytes undergoing comparison coelute. The disadvantages associated with these methods include the cost of reagents, misidentifications because of differences in labeling efficiencies and problems involved in data interpretation of complex mass spectra as to what are the actual $\mathrm{m} / \mathrm{z}$ "pairs" being compared.

Direct analysis of each sample allows a statistically significant sample size to be analyzed for both the control and cancer populations. Individual samples can subsequently be compared to each other, to population averages or population averages can be compared to one another. This direct analysis is made possible by the sample normalization for intensity and chromatographic alignment to template algorithms utilized here. This normalization is required because reproducibility in splitter-based nano LC systems is compromised by changes in column backpressure relative to the splitter backpressure.

Regardless of the methodology employed, the development of any relevant biomarker goes through several phases. These phases have recently been reviewed and include biomarker discovery, evaluation, identification, determination of biological relevance, development of a clinical assay and ultimately a population screen that will reduce the burden of disease on the target population $[16,18]$. These biomarkers will be characterized by a very high specificity (low false-positive rate) in order to detect tumors in the early stages of their development. 
A predictive biomarker(s) for clinical use will likely reside in serum or plasma enabling an assay for early stage cancer screening. Because of the prevalence of abundant proteins (e.g., albumin, immunoglobulins, transferrin, alpha-1-antitrypsin, fibrinogen, etc.) techniques must be utilized which can parse through the abundant proteins/peptides which can circulate at 30$50 \mathrm{mg} / \mathrm{ml}$ (albumin) to find the biomarkers which will be found in concentrations several orders of magnitude lower. The mass action of these abundant proteins can overload chromatographic columns precluding the detection of potential biomarkers that circulate at much lower concentrations. Most commercial kits for removing these abundant proteins utilize some form of affinity (e.g., Cibacron Blue or anti-human serum albumin antibodies) or ion exchange chromatography to remove albumin. Alternatively, size exclusion could be appropriately utilized to separate some of these more abundant proteins that all tend to have relatively high masses from a low molecular weight fraction. Since it is just as likely that the molecular weight of biomarkers shed by tumor tissue will be in the low molecular weight fraction, we chose to analyze serum from stage III/IV ovarian cancer patients and age matched controls for the presence of ovarian cancer biomarkers in a low molecular weight fraction. The protocols and bioinformatics necessary for our differential proteomics analysis of the low molecular weight serum proteome will be discussed.

\section{Experimental}

\subsection{Sample preparation}

Individual or pooled serum from patients with stage III/IV ovarian cancer or age-matched controls $(200 \mu \mathrm{l})$ was diluted 1:1 with water and fractionated on a $10 \mathrm{kDa}$ or $50 \mathrm{kDa}$ microconcentrator (Vivaspin 500, 10,000 MWCO, VivaScience, Carlsbad, CA, USA; Microcon YM-50, 50,000 MWCO, Millipore Corp., Bedford, MA, USA) for 20 minutes at $12,000 \times$ g). An additional $200 \mu \mathrm{l}$ of water was added to the retentate and vortexed briefly prior to separation (20 minutes, 12,000 $\times \mathrm{g})$. The filtrate was lyophilized in a vacuum centrifuge (3-4 hours) and the residue dissolved in $200 \mu \mathrm{l}$ of solvent $\left(95 / 5 \mathrm{H}_{2} \mathrm{O} /\right.$ Acetonitrile, with $0.2 \% \mathrm{H}_{2} \mathrm{CO}_{2}$, $0.1 \%$ Trifluoroacetic acid and $0.001 \%$ Zwittergent $3-$ 16 (Calbiochem, LaJolla, CA, USA)). The injection volume was $25 \mu 1$.

\subsection{Chromatography}

Nano LC columns were packed with Magic C18 (5 $\mu \mathrm{m}, 300 \AA$, Michrom Bioresources, Auburn, CA, USA). All columns were $75 \mu \mathrm{m}$ I.D. and were either packed into a PicoFrit column (360/75 $\mu \mathrm{m}$ OD/ID with a $15 \mu \mathrm{m}$ tip, New Objective, Inc., Cambridge, MA, USA) or a Nanovolume column end fitting (VICI Valco Instruments Co. Inc., Houston, TX, USA) inserted into a $1 / 16$ " ( $1 / 32$ " reducing union ending in a PicoTip emitter (360/20 $\mu \mathrm{m}$ OD/ID with a $10 \mu \mathrm{m}$ tip, New Objective, Inc., Cambridge, MA, USA). All packed columns were $3.5-4.0 \mathrm{~cm}$ long. A $0.3 \times 5 \mathrm{~mm}$ trap packed with Magic C8 $(5 \mu \mathrm{m}, 300 \AA$, Michrom Bioresources, Auburn, CA, USA) was plumbed into a 0.006" 10-port valve (VICI Valco Instruments Co. Inc., Houston, TX, USA). An isocratic loading pump (30 $\mu \mathrm{l} / \mathrm{min}$, 95/5 $\mathrm{H}_{2} \mathrm{O} /$ Acetonitrile, with $0.2 \% \mathrm{H}_{2} \mathrm{CO}_{2}, 0.005 \%$ Trifluoroacetic acid) was utilized to load sample on the trap. The trap was washed for three minutes with loading solvent at which time the valve switches to position $\mathrm{B}$ and bound material is eluted onto the nanoLC column with an organic gradient at $\sim 500 \mathrm{nl} / \mathrm{min}$ (A: 95/5 $\mathrm{H}_{2} \mathrm{O} / \mathrm{ACN}, 0.2 \%$ Formic, $0.005 \%$ TFA; B: 5/95 $\mathrm{H}_{2} \mathrm{O} / \mathrm{ACN}, 0.2 \%$ Formic, $0.005 \%$ TFA; Gradient: 0$14 \mathrm{~min}$. 0-65\% B; 14-16 min. 65-75\% B; 16-18 min. $75-95 \% \mathrm{~B} ; 18-20 \mathrm{~min}$. $95 \% \mathrm{~B} ; 20-22 \mathrm{~min} .95-0 \%$ B; 23 min. STOP) from two Shimadzu LC-10ADvp pumps with a SCL-10Avp controller (Shimadzu Scientific, Columbia, MD, USA).

\subsection{Mass Spectrometry}

A time-of-flight mass spectrometer (Micromass LCT, Waters/Micromass, Milford, MA, USA) was utilized to acquire spectra for cancer and control low MW fractions over the $150-2500 \mathrm{~m} / \mathrm{z}$ range. This data was utilized to differentiate control and cancer proteomes. Several of these same samples were also analyzed on a Fourier transform ion-cyclotron-resonance mass spectrometer (IonSpec, Irvine, CA, USA) equipped with a 7 Tesla actively-shielded superconducting magnet (Cryomagnetics, Oak Ridge, TN) and a modified source (Analytica of Branford, Branford, CT) with a heated metal capillary and a new dual ESI source [17]. Data from each instrument was converted into ASCII format for bioinformatic analysis.

A quadrupole-time-of-flight mass spectrometer (QTOF II, Waters/Micromass, Milford, MA, USA) performed MS/MS analysis on a putative biomarker; that being a doubly charged precursor ion that differen- 

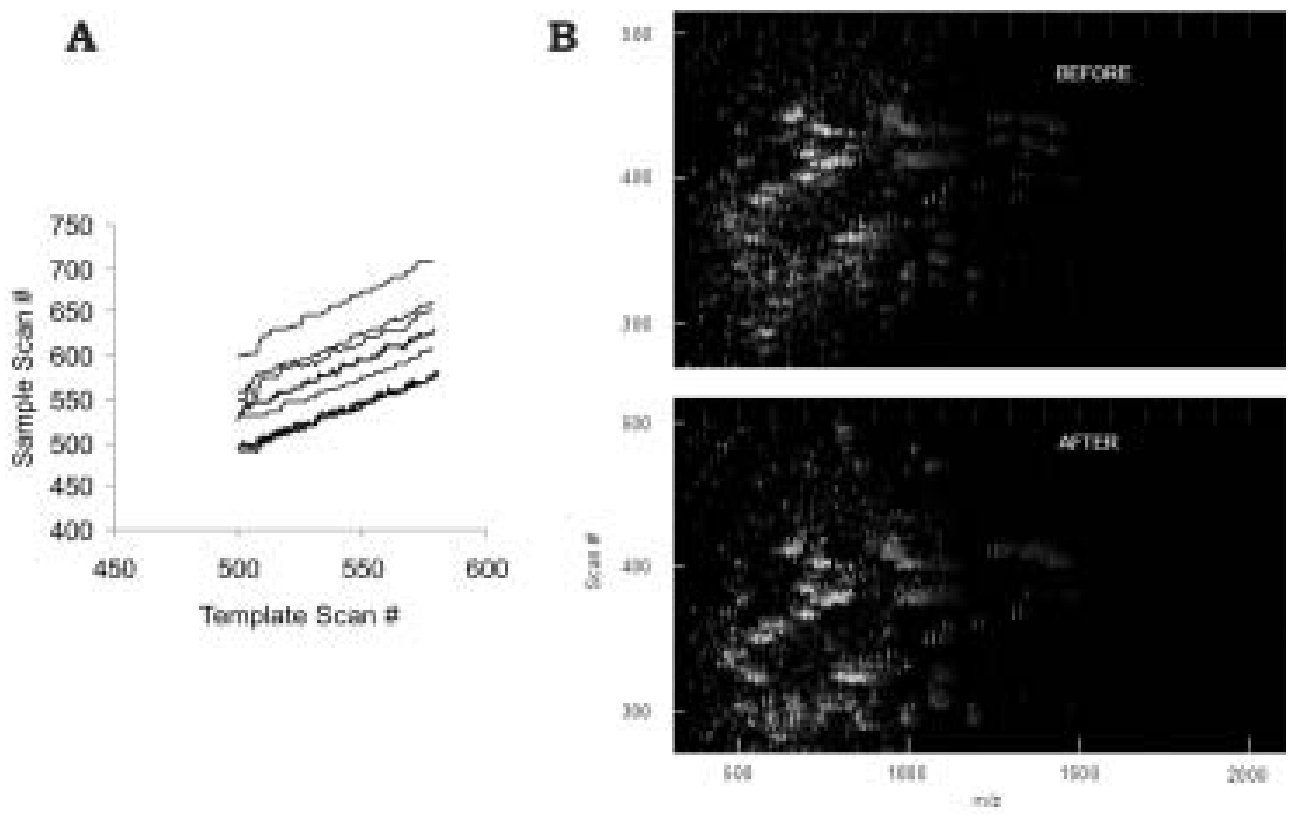

Fig. 1. A) The plot of the chromatographic alignment required for eight injections of the same sample relative to a ninth injection of the same sample that is regarded as the "template". The scan number of the template is recorded on the x-axis and the scan number of each sample that would correspond to the same scan of the template is recorded in the y-axis. B) Chromatographic alignment is necessary due to differences in pressure between the splitter and nanoLC column. Improvement in the chromatographic alignment after normalization is seen in the "after" panel for one sample (red) and a template sample (green) (colour can be viewed in the online version).

tiated the two populations using the bioinformatics algorithms presented (vide infra). Resulting spectra were searched against a human non-redundant protein database for identification using both SEQUEST (Thermofinnigan, San Jose, CA, USA) and MASCOT (Matrix Science Ltd. London, UK) search software.

\subsection{Bioinformatic analysis}

Conversion of LCT spectra to raw ASCII format was performed using the Masslynx/Databridge software (Waters/Micromass, Milford, MA, USA) producing a data-file for each sample. A custom software program analyzed the scan number (retention time), $\mathrm{m} / \mathrm{z}$ value and the ion count (intensity) for each data set. The maximum allowed $\mathrm{m} / \mathrm{z}$ value was 2,200 and the maximum number of scans in the chromatographic dimension was 2,000. A threshold of five ion counts must be exceeded to be included in the analysis.

The original LCT raw data contained four decimal places. Binning intensities to the nearest $0.1 \mathrm{Da}$ collapsed this data producing 10 bins for each Dalton. There were 22,000 possible bins for each scan after binning the $m / z$ dimension. Accumulated intensities were stored in a 22,000 $\times 2,000$ data-table for each sample. The dataset from the first sample was chosen as the template and stored into a separate template-table. The template sample was selected by computing correlation coefficients between all possible pairs of samples and choosing the sample that correlated the best with all other samples. All samples were then normalized for intensity against the designated template by multiplying each intensity value at each $\mathrm{m} / \mathrm{z}$ for each sample by $1 /$ slope where the slope is obtained from the least squares fit line between the sample and the template sample.

The data from all the samples were next aligned with the template sample on the chromatographic axis. Intensities at each $\mathrm{m} / \mathrm{z}$ value were compared to the template data set by shifting sample scans -150 to +150 scan-shifts relative to the template. The correlation coefficients between the sample and template data were then calculated exhaustively for all the shifts. The shift with the best correlation coefficient was then determined and imposed to the sample data. Data for each sample were aligned to achieve maximum correlation with the template data.

Every 50 scans of each shifted data set was then collapsed into one scan by summing the intensities for each $0.1 \mathrm{Da} \mathrm{m} / \mathrm{z}$ bin. Since ion intensity varies with chromatographic elution the normalization is needed to adjust the intensities at each $\mathrm{m} / \mathrm{z}$ value to the average 


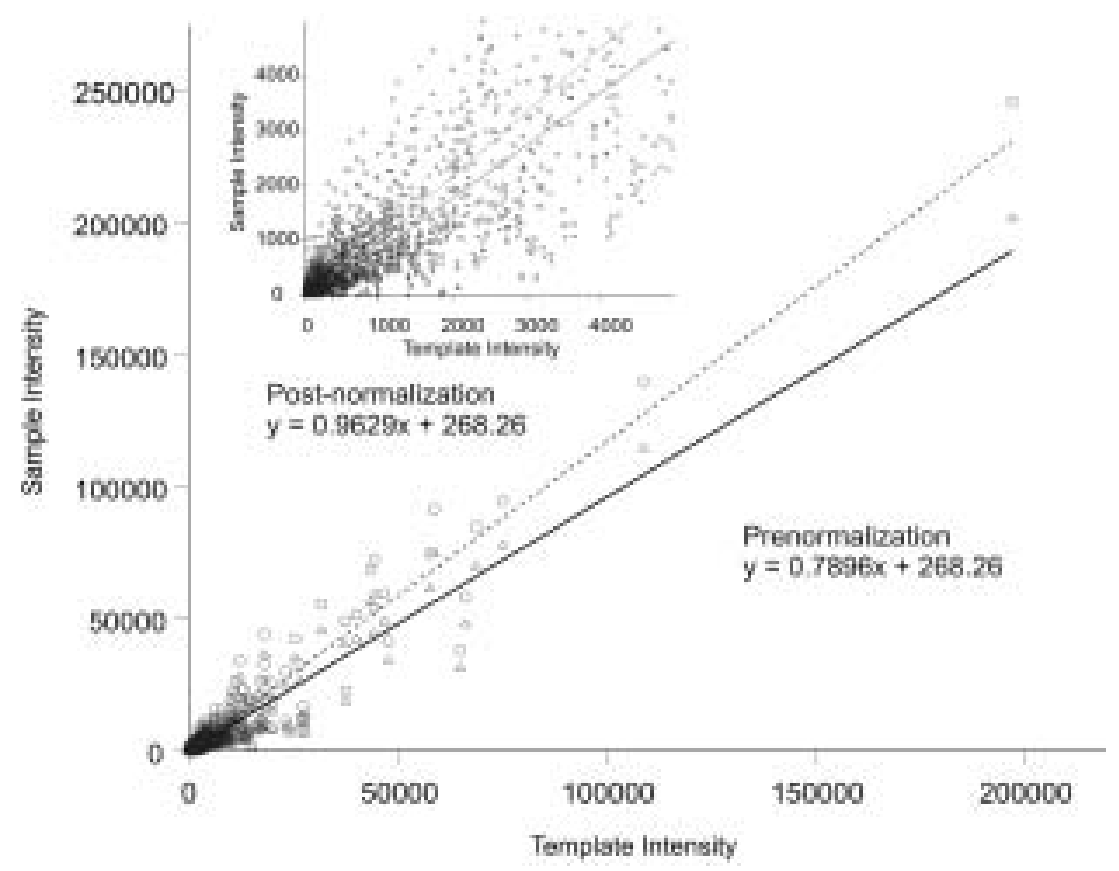

Fig. 2. After chromatographic alignment the intensity of each sample of the control or cancer group is normalized to the intensity of a "template" data set chosen from the same group. The prenormalized and post-normalized data are indicated by $\Delta$ and $\mathrm{O}$ respectively. The slope of the normalized data set approaches unity. The inset is a zoomed look at the values with lower intensity and shows the tremendous number of data points the algorithm must normalize.

intensity of each eluting mass. The intensities of each shifted and collapsed data set was then compared with the intensities of a template sample by calculating the slope of the least squares fit line prepared by plotting all intensities of the template against all the intensities of the sample.

\section{Results}

\subsection{Chromatographic alignment, normalization}

Separations utilizing nanoLC columns frequently have analytes eluting in a 20 second window. Thus, a 30 second shift in retention time can lead to the misidentification of potential biomarkers if a direct comparison between two samples is attempted without some type of alignment on the chromatographic axis (i.e., scan \# or retention time). This need for alignment was determined after analyzing the same sample (pooled stage III/IV ovarian cancer serum) multiple times ( $N=5$ cancers and $N=5$ controls). These shifts in chromatographic retention time can be accounted for by a process we call chromatographic alignment. To validate our chromatographic alignment protocol we ana- lyzed pooled (nine samples) stage III/IV ovarian cancer serums and pooled age matched control (nine samples) serum fractionated by a $10 \mathrm{kDa}$ ultrafiltration membrane. Prior to any data analysis each data set was stripped of data below a set threshold (each $\mathrm{m} / \mathrm{z}$ value must be $\geqslant 5$ counts to be analyzed) and truncated (intensities at each $\mathrm{m} / \mathrm{z}$ value are summed into bins one decimal place wide (i.e., 10 bins/Dalton). Subsequent to the thresholding and truncation, each data set was shifted (by scan \#) until the best correlation coefficient was obtained against a "template" data set for all intensities. Figure 1(A) shows the degree of shifting required for several different injections of the same sample against one sample selected as the template sample. The results of this shifting are shown graphically in Fig. 1(B). The data from a single sample (green) against the template sample (red) is superimposed before and after chromatographic alignment. If two samples were perfectly aligned only a single color would be apparent. The increased agreement between scan number and $\mathrm{m} / \mathrm{z}$ is readily seen in Fig. 1(B).

After comparison of the data generated from the same sample injected multiple times, it became apparent that normalization for intensity was also required. One data set was selected as the "template" data set 


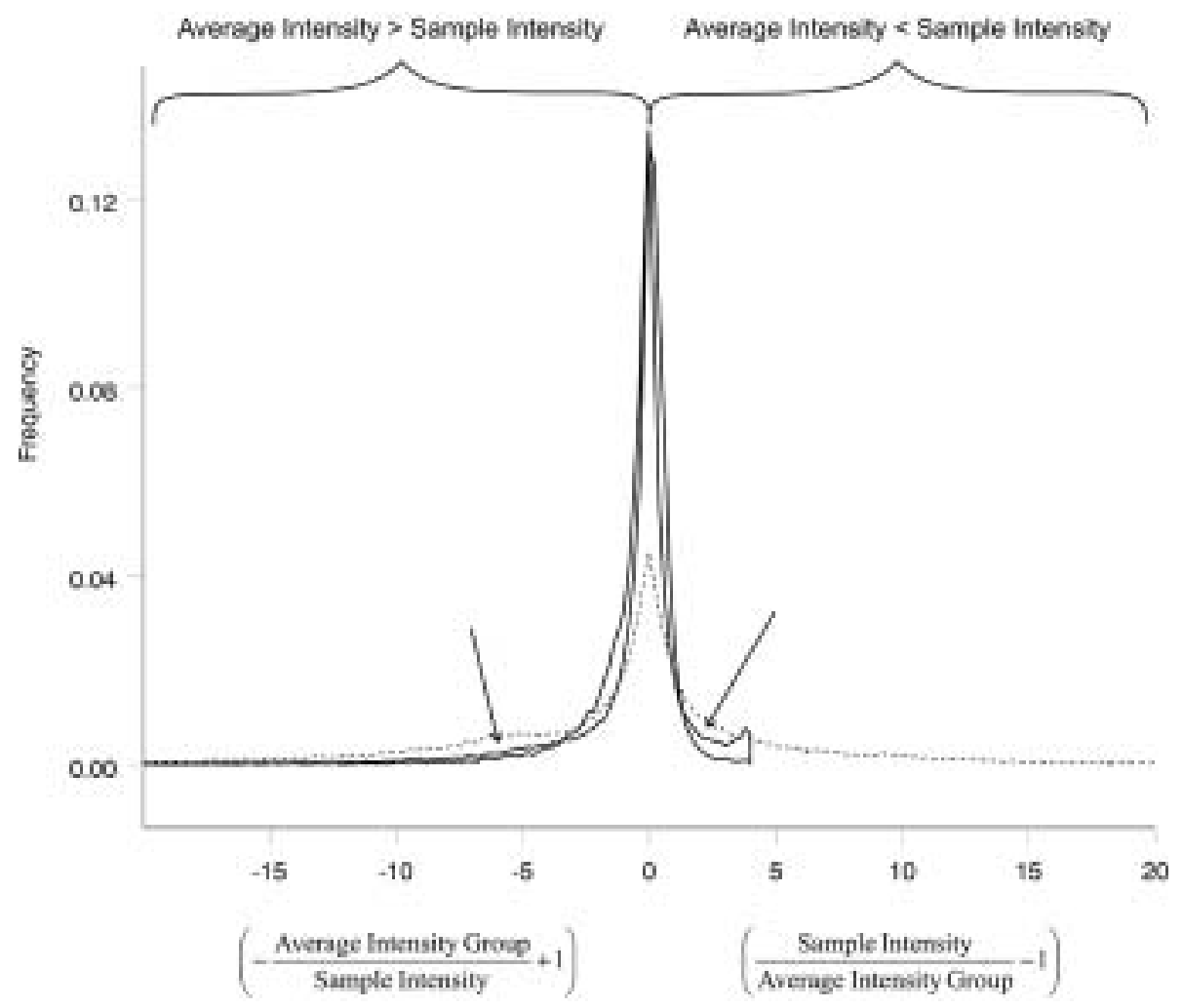

Fig. 3. After the normalization protocols were applied, a plot of frequency of occurrence was generated as the ratio of each group average according to the following protocols: If cancer average $>$ control average the ratio $=\left(-\right.$ cancer average $\left._{\text {control average }}+1\right)$ was plotted. If canceraverage $<$ control $_{\text {average }}$ the ratio $=\left(\right.$ control $_{\text {average }} /$ canceraverage -1$)$ was plotted. To assess the reproducibility of our normalization protocols each normalized control and cancer sample (solid lines) were compared to the normalized control and cancer group averages, respectively. The cancer samples were then compared against the control average and the control samples against the cancer average (dashed line). As expected, the cancer and control groups are mostly identical with the majority of species having close to identical intensities (ratio \pm 1 ). Potential biomarkers to be examined will lie in the area between the dashed curve and the solid curves as indicated by the arrows.

and all other data sets were normalized to the intensity of the "template" data. Figure 2 shows the correlation between one data set and the "template" data set. A slope of unity would indicate perfect agreement between sample and template. The reasonable assumption that most low molecular weight species do not change in concentration between control and the cancer groups allows us to carry out this straightforward normalization procedure. As an example, the slope prior to normalization was 0.79 and post-normalization it increases to 0.96 .

\subsection{Reproducibility}

Once the data has been normalized for chromatographic alignment and intensity, every 50 scans of each data set was collapsed into one scan by summing the intensities of fifty scans at each 0.1 Da increment. After summing the 50 scans, a differential comparison can be made. For this analysis, each normalized control and cancer sample was compared to the normalized control and cancer group averages, respectively. The cancer samples were then compared against the control average and the control samples against the cancer average at each $\mathrm{m} / \mathrm{z}$ value. Depending on whether or not the average intensity is less than or greater than the sample intensity we used the following two equations to plot the results:

For Average Intensity $>$ Sample Intensity Ratio $=$ $\left(-\frac{\text { Average Intensity Group }}{\text { Sample Intensity }}\right)+1$

For Average Intensity $<$ Sample Intensity Ratio $=$ $\left(\frac{\text { Sample Intensity }}{\text { Average Intensity Group }}-1\right)$

A ratio of zero indicates no difference between cancer and control. Figure 3 indicates the very good reproducibility obtained for the normalized data. The majority of species in both the cancer and control have a corresponding intensity that is centered about zero or 

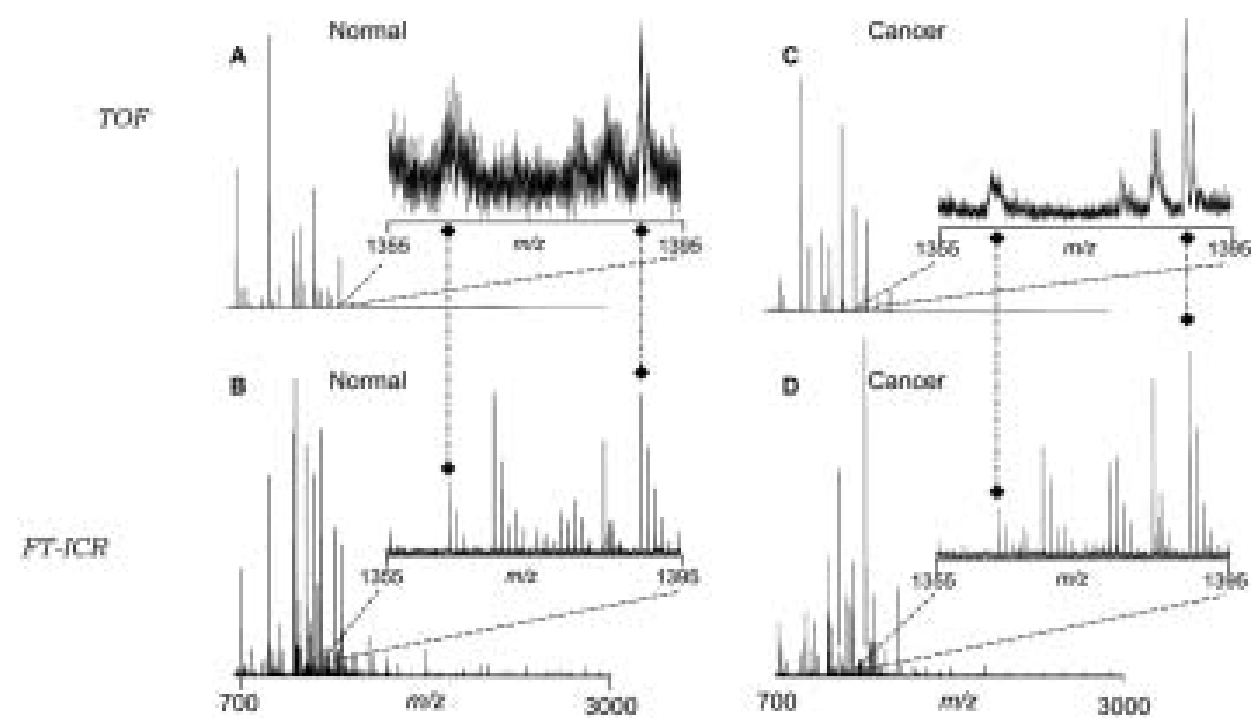

Fig. 4. The normalization protocols were applied to nine cancer and nine control samples for biomarker discovery. Differences and similarities exist, as would be expected, between cancer and control samples. Additionally, the FT-ICR data not only corroborates the TOF data, but also is several-fold richer in new data. The better resolving power available with the FT-ICR is readily apparent in the spectra shown.

close to zero (indicating no difference). Thus, we see the highest frequency at or close to zero indicating that the majority of $\mathrm{m} / \mathrm{z}$ values of a sample have intensities identical to group averages of the same group (e.g., control sample compared to control group average, etc.). Likewise, when each sample is compared to the opposite group average (e.g., control sample compared to cancer group average) the majority of $\mathrm{m} / \mathrm{z}$ values are identical. Potential biomarkers to be examined will lie in the area between the solid curves (within group) and the dotted curve (between group) as indicated by the arrows.

\subsection{Analysis of stage III/stage IV and age-matched controls}

To apply these results to the discovery of ovarian cancer biomarkers, nine cancer and nine control samples were individually analyzed. Differences and similarities exist as would be expected between cancer and control samples. Additionally, when the same samples were examined with the FT-ICR (Fig. 4) the spectra not only corroborate the TOF data, but also are several-fold richer in new data. We also saw enrichment in low molecular weight species when we increased the molecular weight cut-off filter from 10 to $50 \mathrm{kDa}$ (Fig. 5).

The above protocols were then applied to seventeen samples normalized to one sample (nine cancer and nine control). The frequency of occurrence comparison between control and cancer samples as a func- tion of ratio is plotted in Fig. 6. Again, the majority of species are centered around zero indicating no difference in intensity and thus most species are present in both cancer and control samples equivalently. Potential biomarkers will lie outside this range. There were over 15 molecular entities that differed by more than a factor of 5. One such candidate biomarker is shown in the inset of Fig. 6. This species $(\mathrm{m} / \mathrm{z}=$ 1,582 ) has a cancer-to-control intensity ratio of 6.3 and since it is a doubly charged ion a nominal molecular weight of 3,160 Da. The receiver-operating characteristic (ROC) plot generated from this data is also shown as an inset of Fig. $6(N=18)$. Calculating the sensitivity and specificity of this particular mass for each sample value as a cut-off generates an ROC plot as shown with an area under the curve of 0.815 . Minimizing $\sqrt{(1-\text { sensitivity })^{2}+(1-\text { specificity })^{2}}$ yields a sensitivity of $70 \%$ and a specificity of $100 \%$ for the $1582 \mathrm{Da}$ species. It must be kept in mind that these results were generated with a limited number ( 9 normal and 9 control) of stage III/IV serum that constituted $50 \%$ of our samples not $0.01 \%$ as the actual ovarian cancer incidence rate requires.

When this putative ovarian cancer biomarker was subjected to MS/MS analysis the tandem mass spectrum of Fig. 7 suggested at least one site of phosphorylation as evidenced by losses of 98 mass units, characteristic of neutral loss of $\mathrm{H}_{3} \mathrm{PO}_{4}$ from phosphorylated serine or threonine [6]. The MS/MS spectrum was searched against the NCBI nr human pro- 

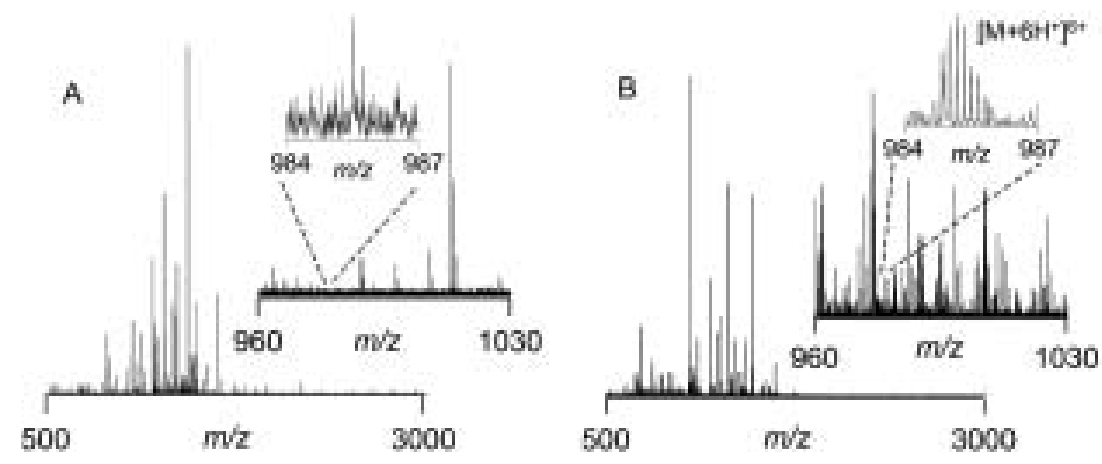

Fig. 5. Comparison of the filtrate obtained using a $10 \mathrm{kDa}$ (Fig. 5(A)) versus a $50 \mathrm{kDa}$ (Fig. 5(B)) molecular weight cutoff filter. The lower molecular weight species were significantly enriched using the $50 \mathrm{kDa}$ filter.

tein and EST databases while allowing for phosphorylation modifications. When the subsequence ADSGEGD was searched, nine entries related to fibrinogen-A were returned. A MASCOT sequence tag search yielded the sequence VCLVLSVVGTAWTADSGEGDFLAEGGGVRG, from fibrinogen $\mathrm{A}$, with three phosphorylation sites. Manual interpretation suggests the following partial sequence: $\left(\mathrm{N}_{\text {term }} \ldots\right.$ ADpSGEGDFLAEGGGVR . .- $\mathrm{C}_{\text {term }}$ ). This sequence corresponds to fibrinopeptide A with one phosphorylated serine [3]. Thrombin cleaves fibrinogen A into fibrin (the clotting protein) and fibrinopeptide-A. The mass of fibrinopeptide-A is $1615.7 \mathrm{Da}$ while our doubly charged precursor ion utilized for MS/MS analysis was $1582 \mathrm{Da}$ with a nominal molecular weight of 3,160 Da. Clearly, a partial identity of the putative ovarian cancer biomarker has been made, but its clinical utility and underlying biology remains to be elucidated.

\section{Discussion}

Pepe et al. and Muddiman et al. have recently proposed roadmaps for biomarker discovery and validation for the early detection of cancer $[16,18]$. These roadmaps both entail a discovery or exploratory phase, clinical assay development of an identified biomarker and population studies to obtain positive predictive values that are at acceptable levels. The ultimate goal of any biomarker discovery program is to reduce the burden of disease on the target population. Although the clinical assay development and population studies may follow a more traditional route, the discovery or exploratory phase has been greatly impacted by the proteomics era.

Previous success in identifying biomarkers for ovarian cancer have involved the co-screening of mono- clonal antibodies raised against cancer tumor preparations and screened against the tumor preparation and a non-cancerous control tissue/culture. Clones that were positive for the cancer preparation and negative for the control were utilized as screening agents without any a priori knowledge regarding the actual antigen. The CA-125 ovarian cancer marker was identified in this manner and has been utilized for eighteen years without any knowledge of the antigens identity [26]. A paradigm shift has occurred with the advent of differential proteomics techniques that are capable of comparing tissue or associated fluids (e.g. plasma, cerebrospinal fluid) from cancer and control samples directly. These techniques are also capable of identifying the protein biomarkers based on an accurate mass tag or sequence tag $[5,14]$.

The differential proteomics toolbox currently includes isotope-coded affinity tags (ICAT), global internal standardization technology (GIST), surfaceenhanced laser desorption and ionization time-of-flight (SELDI-TOF) and multidimensional protein identification technology (MudPIT) $[4,9,19,23,24]$.

In this paper we have shown that chromatographic alignment and intensity normalization can successfully be utilized to identify candidate ovarian cancer biomarkers with significant biological relevance.

Regardless of the method selected, prior to elucidating candidate biomarkers, the mass action effects of the abundant serum proteins must be successfully dealt with. We initially experimented with liquid-liquid extraction and/or protein precipitation with trichloroacetic acid to separate a fraction of the serum proteome from the abundant immunoglobulins and albumins. Our best results were obtained by physically separating the low molecular weight fraction from the high molecular weight fraction with a molecular weight cut-off filter. Figures 4 and 5 indicate the de- 


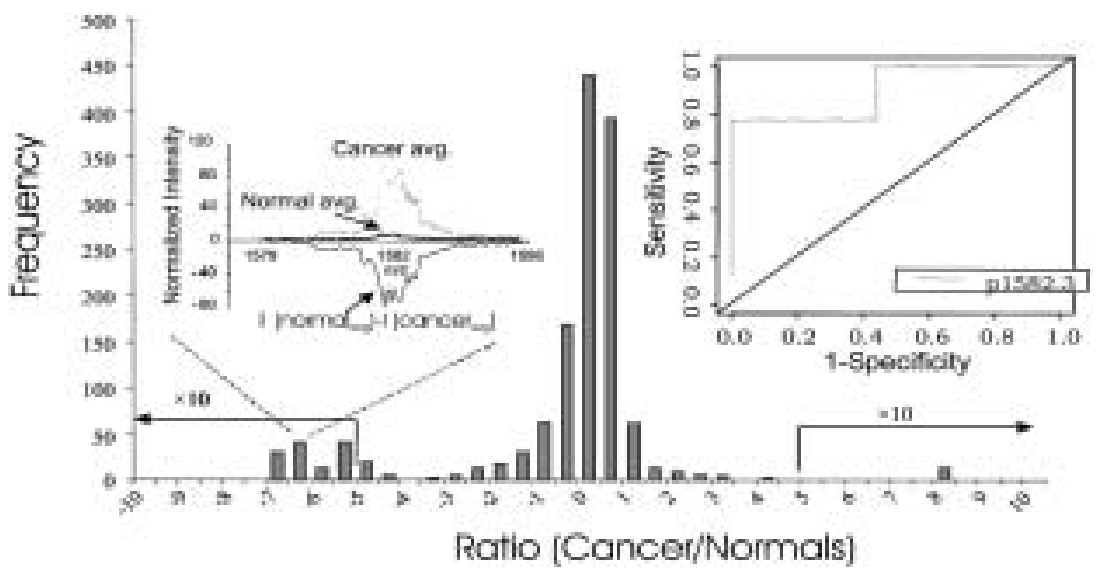

Fig. 6. The plot indicates the frequency at which differences occur when cancer versus control is examined (see Fig. 3 for details). The inset shows the ESI-TOF data of a potential marker whose cancer-to-control intensity ratio was $6.3(\mathrm{~m} / \mathrm{z}=1582)$. The other inset shows the ROC curve generated from the $m / z=1582$ data $(N=18)$.

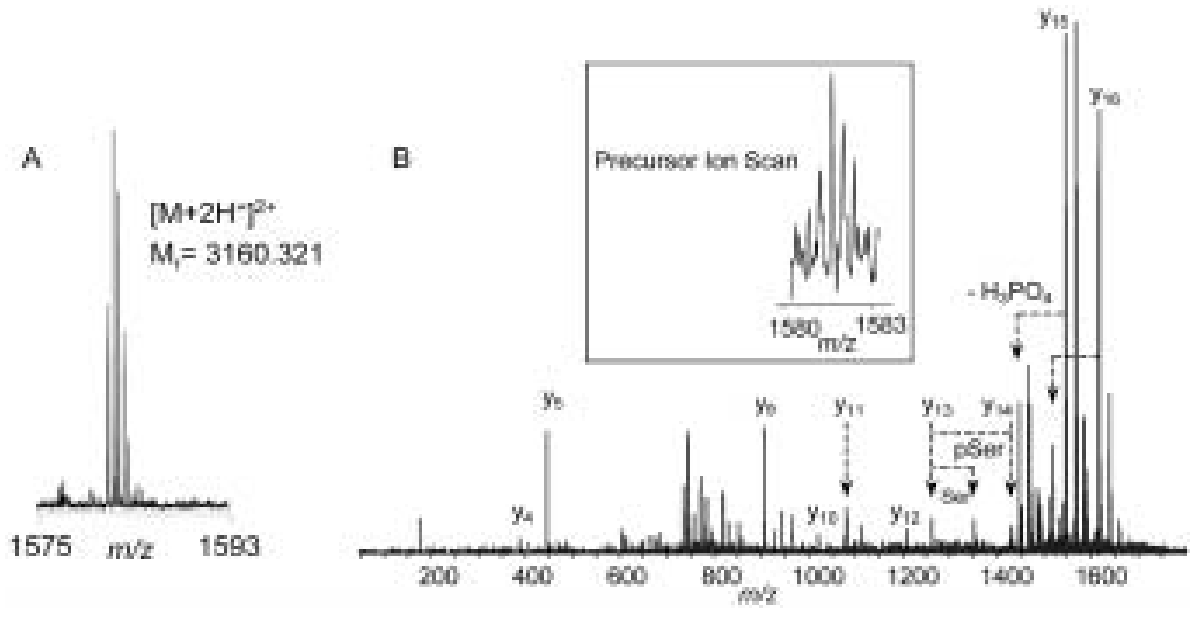

Fig. 7. Identification of putative ovarian cancer biomarker with $\mathrm{Mr}=3160.321$ showing: A) the superior mass accuracy, resolving power and signal-to-noise ratio of FT-ICR data versus 1D-LC-MS/MS experiment on a Q-Tof (inset of B), and B) the MS/MS spectrum showing y-type fragment ions which support the C-terminal partial peptide sequence of . . . . . ADpSGEGDFLAEGGGVR, homologous with fibrinopeptide-A.

gree of heterogeneity of this low molecular weight proteome. When comparing a $10 \mathrm{kDa}$ (Fig. 5(A)) and $50 \mathrm{kDa}$ (Fig. 5(B)) cut-off filter, the $50 \mathrm{kDa}$ filtered sample showed a marked increase in abundance with the same species found in the $10 \mathrm{kDa}$ filtrate which is attributed to higher recovery and, as expected, additional species were found that were not found in the $10 \mathrm{kDa}$ filtrate (Fig. 5). No evidence for the deterioration of nanoLC column performance that would be associated with the presence of albumin or other abundant proteins was seen during the course of our analyses. Based on the results presented in this work, the low molecular weight fraction is rich in potential biomarkers, is easily prepared and resolves a major issue in serum/plasma analysis; namely, the removal/accommodation of the abundant proteins that can effectively mask the detection and analysis of low abundance proteins/peptides. These results compare favorably with the results of Tirumalai et al. who found the $<30 \mathrm{kDa}$ serum filtrate to be rich in proteins associated with cell signaling, necrosis, apoptosis and hemolysis [21]. Additionally, this group found that the protein/peptide content of the $<30 \mathrm{kDa}$ filtrate could be enhanced by addition of solvents that disrupt protein-protein interactions [21]. Our samples did not benefit from the enhancement provided by addition of denaturants, but were still rich in hetero geneity.

We found that utilizing FT-ICR for detection also 
increases the sensitivity, dynamic range, and resolving power of potential biomarkers in the low molecular weight serum fraction (Fig. 4). Our current ICR instrumentation requires a $6-10$ second dead time associated with the transfer of the large data sets from the ICR digitizer and onto the computer. Because analytes are eluting in a 20 second wide peak, our current FT-ICR is incapable of accommodating the quick elution profiles of the nanoLC separations in order to make direct comparisons between samples (i.e., there is significant risk of blind spots being created). New generation data stations are being installed to remove this barrier. Our initial data indicates that FT-ICR will be the detector of choice as soon as the data transfer rate can be increased to an acceptable level.

Analysis of our data indicates that the majority of $\mathrm{m} / \mathrm{z}$ values are nearly identical in abundance as would be expected for the majority of serum/plasma components (Figs 3 and 6). Thus, where the intensity at a particular $m / z$ value is identical in both control and cancer sample, the frequency plotted in Figs 3 and 6 yields a value of zero on the $\mathrm{x}$-axis. As expected, the highest frequency is centered about zero where the intensities are identical.

The search for significant biomarkers occurs where $x \neq 0$. In our comparison of control and cancer samples we identified over 15 molecular entities that differed by more than a factor of 5 and chose to look at one doubly charged $\mathrm{m} / \mathrm{z}$ value $(\mathrm{m} / \mathrm{z}=1582)$ that was statistically significantly different in the cancer sample versus the control samples. Part of the sequence of this potential biomarker was identical to fibrinopeptide-A. Filtering this putative biomarker through a biological relevance filter quickly leads to the observation that hematocrit-independent hyperviscosity is often associated with ovarian cancer $[16,22,27]$. Additionally, fibrinopeptide-A concentrations are significantly correlated to the disease stage of ovarian cancer and are significantly higher compared to controls [7,22].

The fact that our methodology identified a serum component that is known to be elevated in ovarian cancer patients without any prior knowledge illustrates the power and usefulness of this technique for the identification and characterization of cancer biomarkers from serum.

\section{Conclusions}

An algorithm was developed for sample normalization for intensity and chromatographic alignment to a template that allows for the direct comparison of cancer and control proteomes. The protocols developed were utilized to examine the low molecular weight fraction of serum from stage III/IV ovarian cancer patients and age matched controls. Molecular weight cut-off filters effectively separated the abundant serum proteins from a low molecular weight fraction that is rich in potential biomarkers. One potential biomarker was characterized and contains the sequence of fibrinopeptideA. A high degree of biological relevance can be associated with this identified fibrinogen fragment since fibrinopeptide-A concentrations are significantly correlated to the disease stage of ovarian cancer. The protocols outlined here provide the opportunity to identify biomarkers in a generic manner for any disease in which disease and control serum are available.

\section{Acknowledgments}

We are grateful for the financial support of the W.M. Keck Foundation and the Mayo Clinic College of Medicine.

\section{References}

[1] R.C. Bast, Jr., Status of tumor markers in ovarian cancer screening, J Clin Oncol 21 (2003), 200-205.

[2] R.C. Bast, Jr., T.L. Klug, E. St John, E. Jenison, J.M. Niloff, H. Lazarus, R.S. Berkowitz, T. Leavitt, C.T. Griffiths, L. Parker, V.R. Zurawski, Jr. and R.C. Knapp, A radioimmunoassay using a monoclonal antibody to monitor the course of epithelial ovarian cancer, N Engl J Med 309 (1983), 883-887.

[3] C.G. Binnie, J.M. Hettasch, E. Strickland and S.T. Lord, Characterization of purified recombinant fibrinogen: partial phosphorylation of fibrinopeptide A, Biochemistry 32 (1993), 107113.

[4] A. Chakraborty and F.E. Regnier, Global internal standard technology for comparative proteomics, J Chromatogr A 949 (2002), 173-184.

[5] T.P.Conrads, G.A. Anderson, T.D. Veenstra, L. Pasa-Tolic and R.D. Smith, Utility of accurate mass tags for proteome-wide protein identification, Anal Chem 72 (2000), 3349-3354.

[6] S.B. Ficarro, M.L. McCleland, P.T. Stukenberg, D.J. Burke, M.M. Ross, J. Shabanowitz, D.F. Hunt and F.M. White, Phosphoproteome analysis by mass spectrometry and its application to Saccharomyces cerevisiae, Nat Biotechnol 20 (2002), 301-305.

[7] A. Gadducci, U. Baicchi, R. Marrai, B. Del Bravo, P.V. Fosella and V. Facchini, Pretreatment plasma levels of fibrinopeptideA (FPA), D-dimer (DD), and von Willebrand factor (vWF) in patients with ovarian carcinoma, Gynecol Oncol 53 (1994), 352-356.

[8] R.T. Greenlee, M.B. Hill-Harmon, T. Murray and M. Thun, Cancer statistics, 2001, CA Cancer J Clin 51 (2001), 15-36. 
[9] S.P. Gygi, B. Rist, S.A. Gerber, F. Turecek, M.H. Gelb and R. Aebersold, Quantitative analysis of complex protein mixtures using isotope-coded affinity tags, Nat Biotechnol 17 (1999), 994-999.

[10] A. Jemal, A. Thomas, T. Murray and M. Thun, Cancer statistics, 2002, CA Cancer J Clin 52 (2002), 23-47.

[11] C. La Vecchia, Epidemiology of ovarian cancer: a summary review, Eur J Cancer Prev 10 (2001), 125-129.

[12] A.A. Langston and E.A. Ostrander, Hereditary ovarian cancer, Curr Opin Obstet Gynecol 9 (1997), 3-7.

[13] K.O. Lloyd and B.W. Yin, Synthesis and secretion of the ovarian cancer antigen CA 125 by the human cancer cell line NIH:OVCAR-3, Tumour Biol 22 (2001), 77-82.

[14] M. Mann and M. Wilm, Error-tolerant identification of peptides in sequence databases by peptide sequence tags, Anal Chem 66 (1994), 4390-4399.

[15] G.B. Mills, R.C. Bast and S. Srivastava, Future for ovarian cancer screening: Novel markers from emerging technologies of transcriptional profiling and proteomics, J Natl Cancer Inst 93 (2001), 1437-1439.

[16] D. Muddiman, W. Cliby and H. Bergen, in Clinical Laboratory News 29 (2003), 12-16.

[17] A.I. Nepomuceno, D.C. Muddiman, H.R. Bergen, 3rd, J.R. Craighead, M.J. Burke, P.E. Caskey and J.A. Allan, Dual electrospray ionization source for confident generation of accurate mass tags using liquid chromatography Fourier transform ion cyclotron resonance mass spectrometry, Anal Chem 75 (2003), 3079-3086.

[18] M.S. Pepe, R. Etzioni, Z. Feng, J.D. Potter, M.L. Thompson, M. Thronquist, M. Winget and Y. Yasui, Phases of Biomarker Development for Early Detection of Cancer, J Nat Cancer Inst 93 (2001), 1054-1061.
[19] E.F. Petricoin, A.M. Ardekani, B.A. Hitt, P.J. Levine, V.A. Fusaro, S.M. Steinberg, G.B. Mills, C. Simone, D.A. Fishman, E.C. Kohn and L.A. Liotta, Use of proteomic patterns in serum to identify ovarian cancer, Lancet 359 (2002), 572-577.

[20] L.A.G. Ries, M.P. Eisner, C.L. Kosary, B.F. Hankey, B.A. Miller, L. Clegg et al., eds, SEER Cancer Statistics Review, 1975-2000, Bethesda, MD: National Cancer Institute, 2003.

[21] R.S. Tirumalai, K.C. Chan, D.A. Prieto, H.J. Issaq, T.P. Conrads and T.D. Veenstra, Characterization of the low molecular weight human serum proteome, Mol Cell Proteomics 2 (2003), 1096-1103.

[22] G.F. von Tempelhoff, L. Heilmann, G. Hommel, D. Schneider, F. Niemann and H. Zoller, Hyperviscosity syndrome in patients with ovarian carcinoma, Cancer 82 (1998), 1104-1111.

[23] M.P. Washburn, D. Wolters and J.R. Yates, 3rd, Large-scale analysis of the yeast proteome by multidimensional protein identification technology, Nat Biotechnol 19 (2001), 242-247.

[24] D.A. Wolters, M.P. Washburn and J.R. Yates, 3rd, An automated multidimensional protein identification technology for shotgun proteomics, Anal Chem 73 (2001), 5683-5690.

[25] F.J. Xu, Y.H. Yu, B.Y. Li, M. Moradi, S. Elg, C. Lane, L. Carson and S. Ramakrishnan, Development of two new monoclonal antibodies reactive to a surface antigen present on human ovarian epithelial cancer cells, Cancer Res 51 (1991), 4012-4019.

[26] B.W. Yin and K.O. Lloyd, Molecular cloning of the CA125 ovarian cancer antigen: identification as a new mucin, MUC16, J Biol Chem 276 (2001), 27371-27375.

[27] L.R. Zacharski, V.A. Memoli, D.L. Ornstein, S.M. Rousseau, W. Kisiel and B.J. Kudryk, Tumor cell procoagulant and urokinase expression in carcinoma of the ovary, $J$ Natl Cancer Inst 85 (1993), 1225-1230. 


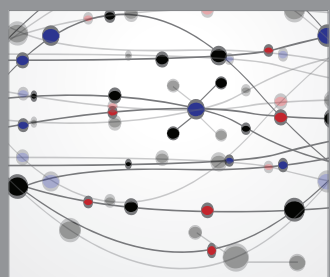

The Scientific World Journal
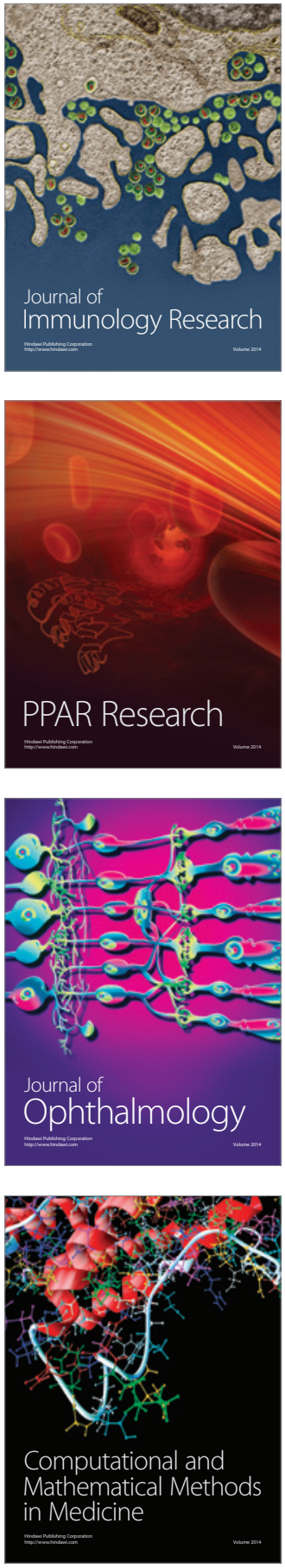

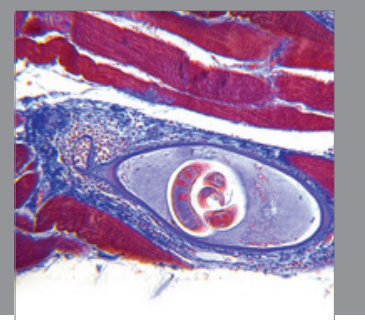

Gastroenterology

Research and Practice
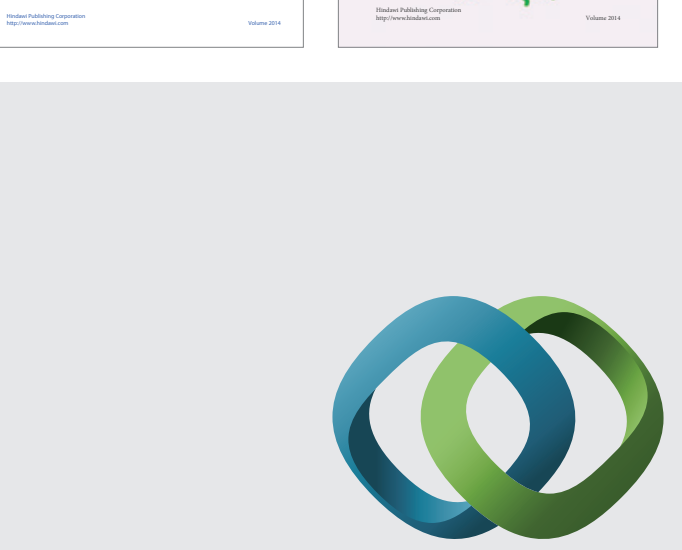

\section{Hindawi}

Submit your manuscripts at

http://www.hindawi.com
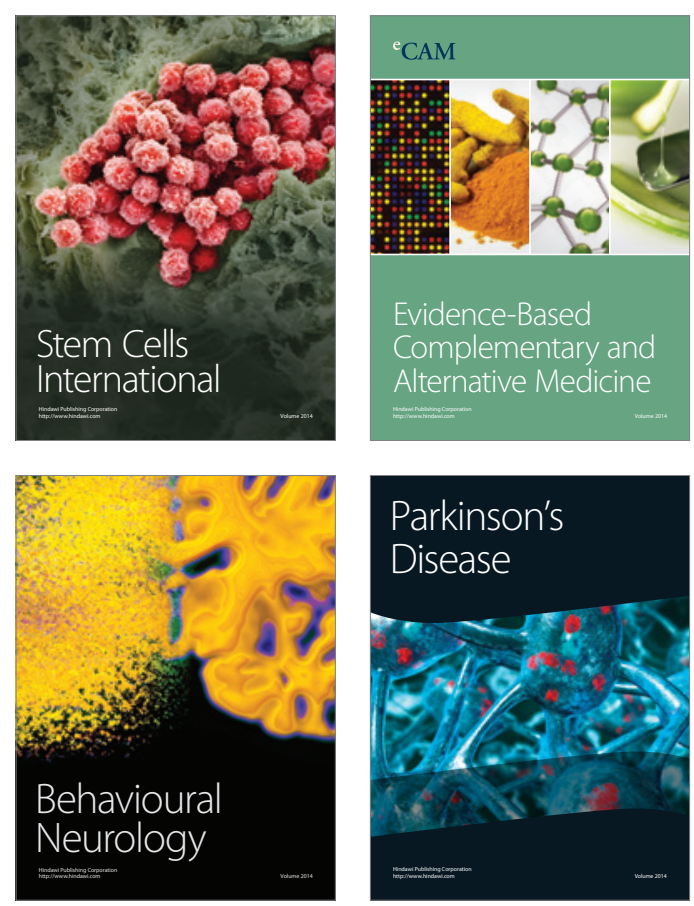

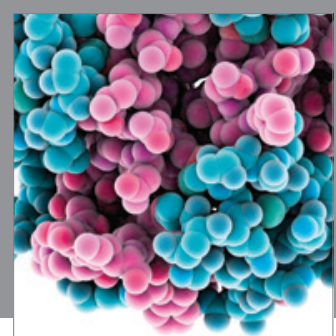

Journal of
Diabetes Research

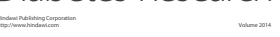

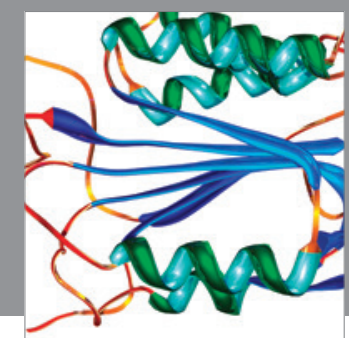

Disease Markers
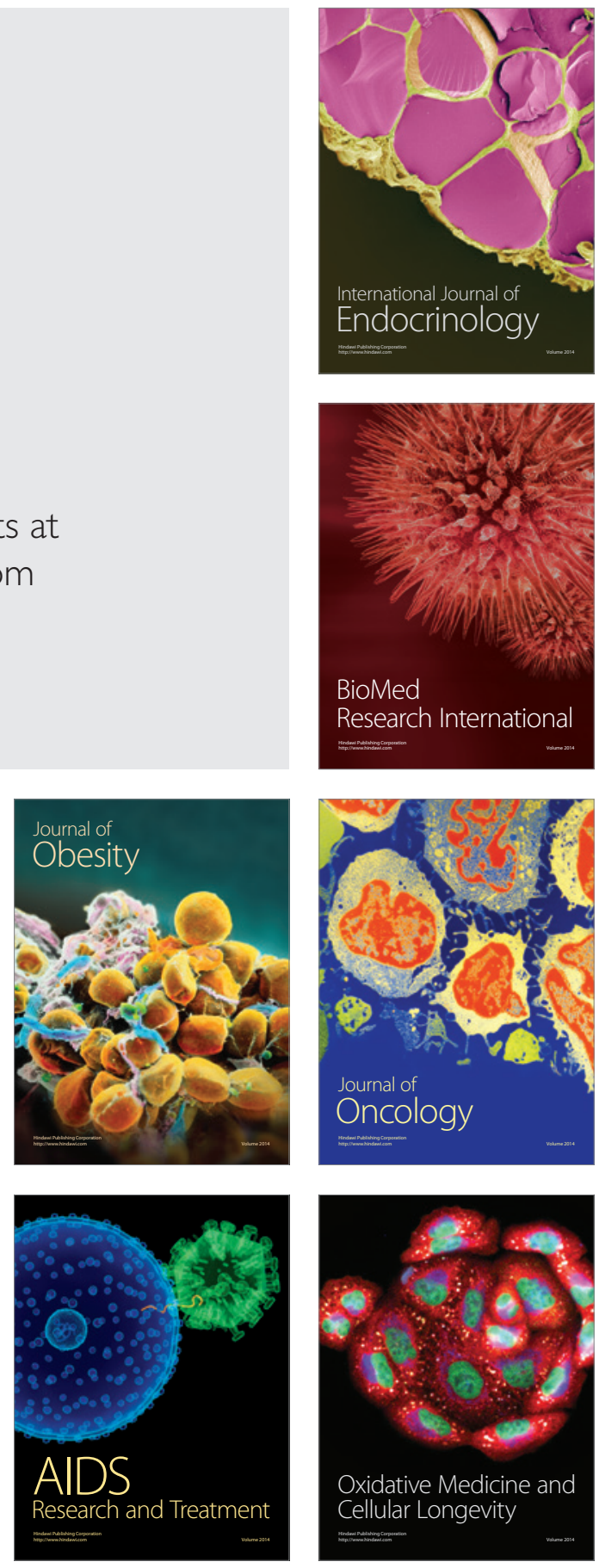